\title{
Pancreatic neuroendocrine tumors with transformation to insulinoma: an unusual presentation of a rare disease
}

\author{
Avital Nahmias ${ }^{1}$, Simona Grozinsky-Glasberg ${ }^{1}$, Asher Salmon ${ }^{2}$ and David J Gross ${ }^{1}$ \\ ${ }^{1}$ Neuroendocrine Tumor Unit, Endocrinology and Metabolism Service ${ }^{2}$ Department of Oncology, Hadassah-Hebrew \\ University Medical Center, Kiryat Hadassah, POB 12000 Jerusalem, 91120, Israel
}

Correspondence should be addressed to A Nahmias

Email

nahmias.avital@gmail.com

\section{Summary}

Approximately $35 \%$ of the pancreatic neuroendocrine tumors (pNETs) are functional, the most common of which is an insulinoma. Rarely can initially nonfunctioning tumor undergo biological transformation to a hormone-secreting tumor with subsequent changes in the clinical picture. We present here three unique patients with long-standing pNETs who developed life-threatening hyperinsulinemic hypoglycemia along with tumor progression. In two of the patients, everolimus (Afinitor) was administered in an attempt to control both tumor growth and hypoglycemia. In two cases everolimus therapy resulted in the abolishment of hypoglycemia and induced significant tumor regression; however these beneficial responses were transient. These cases highlight the exceptional ability of pNETs to change biological behavior in parallel with disease progression. Our experience concurs with recently published studies demonstrating the utility of everolimus for the control of both hypoglycemia and tumor progression.

\section{Learning points:}

- Nonfunctional pNET can gain new features such as insulin secretion with related morbidity.

- Gain of function in a previously nonfunctional pNET signifies tumor progression and is usually associated with poor prognosis.

- Everolimus proved to be a viable treatment for hypoglycemia in insulinoma patients and was also proven highly effective in the patients presented here.

- As disease progresses, the effect of everolimus on hypoglycemia wanes. We report for the first time the development of hypoglycemia during everolimus treatment.

\section{Background}

Pancreatic neuroendocrine tumors (pNETs) present with a heterogeneous clinical behavior (1). The majority of pNETs have an indolent course at early stage, but patients will eventually develop symptoms of tumor progression or hormone secretion that usually requires medical intervention (1). Based on their endocrine secretion, pNETs may be divided into functioning or nonfunctioning tumors. Nonfunctioning tumors comprise about $65 \%$ of
pNETs, and most of them have metastasized at diagnosis (2). Rarely have these tumors been reported to be able to change their phenotype into functioning tumors; however, the mechanism for this acquired switch in the tumor secretory ability is still unclear (3) (4). Current therapeutic options for metastatic disease are limited. The mTOR signaling pathway plays a key role in cell growth, protein translation, autophagy, and cell metabolism (5). 
Evidence linking mTOR signaling to the pathogenesis of pNETs arises from studies of genetic cancer syndromes associated with pNETs, such as tuberous sclerosis, MEN1, and neurofibromatosis. Furthermore, mTOR abnormalities have been correlated with more aggressive tumors. Everolimus inhibits mTOR and was found to be effective in patients with low to intermediate grade, unresectable, or metastatic pNETs that have progressed on prior therapies (6). Everolimus has been shown to affect glycemic control and was utilized to resolve hypoglycemia in insulinoma patients (7); this effect was not attributed to a reduction in tumor size (7) (8). In this report we will present a small series of three patients with metastatic pNET, initially nonfunctioning, which has evolved to insulinoma. In two of the cases everolimus was used to treat both tumor growth and hypoglycemic events; in the third case, hyperinsulinemic-associated hypoglycaemia surprisingly developed during treatment with everolimus.

\section{Case presentation}

\section{Case 1}

A 45-year-old woman presented with abdominal pain, diarrhea, and weight loss.

Investigation Subsequent investigation revealed a mass located in the head of the pancreas with a diameter

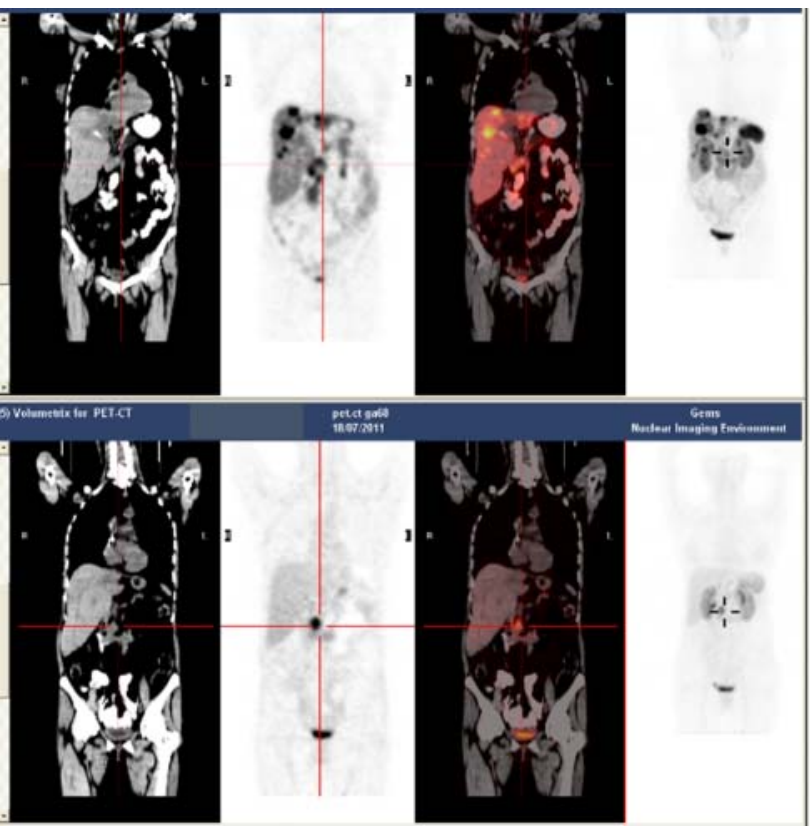

of $2.5 \times 3 \mathrm{~cm}$, with secondary hepatic lesions and a well-differentiated grade 2 NET (KI-67 proliferation index of 5-7\%) was diagnosed on pathology. A somatostatin receptor imaging with ${ }^{68}$ Gallium-DOTATATE PET-CT showed high uptake by the pancreatic and hepatic masses (Fig. 1).

Treatment The patient was initially treated with Somatuline Autogel (lanreotide Autogel, Ipsen) $30 \mathrm{mg}$ every 2 weeks. Subsequently, due to progressive disease, the patient was treated with peptide-receptor radionuclide therapy (PRRT) ${ }^{90}$ Yttrium-DOTATOC with disease stabilization.

Outcome and follow-up Five months later, the patient presented with severe episodes of hypoglycemia with serum glucose levels decreased to $1.6 \mathrm{mmol} / \mathrm{l}$ (normal range 4-6 mmol/l), together with inappropriately high levels of serum insulin $(2021 \mathrm{pmol} / \mathrm{l}$, normal range $=22-180)$ and C-peptide $(2876 \mathrm{pmol} / \mathrm{l}$, normal range $=268-1274)$. A second biopsy from a liver metastasis revealed a higher proliferation index of $20 \%$. Subsequent treatment with everolimus followed by streptozocin, dischlorodiammine platinum (DDP), and 5 fluorouracil (5-FU) resulted in resolution of the hypoglycemia and marked regression of the hepatic lesions. However, 18 months later the disease progressed, and despite the administration of salvage
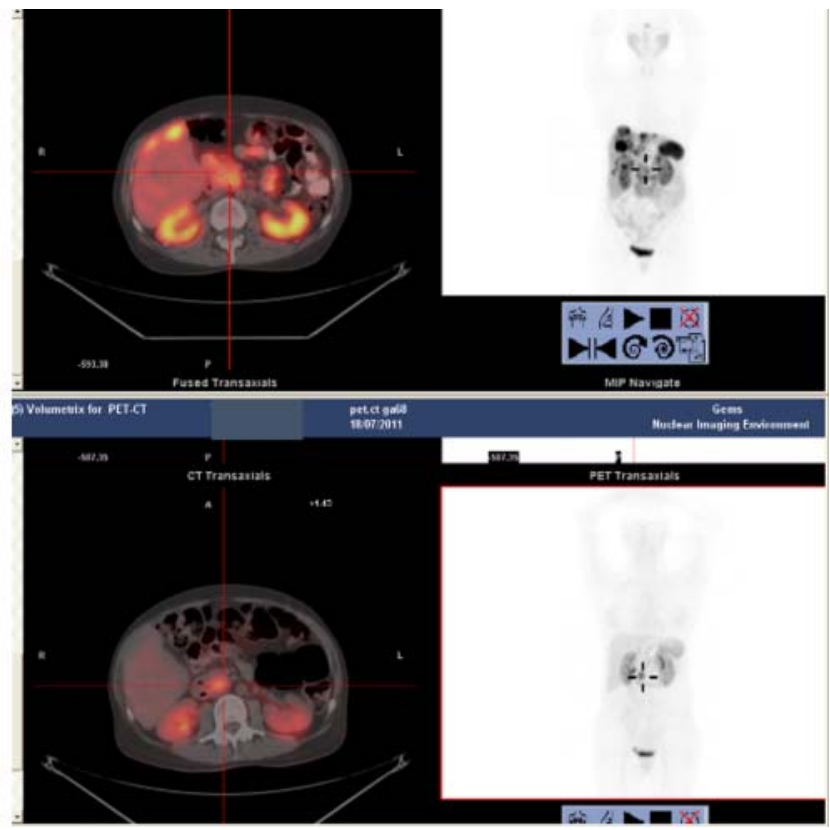

Figure 1

${ }^{68}$ Gallium-DOTATATE PET-CT of the first patient showing high uptake by the pancreatic and hepatic masses. 
PRRT, the patient died due to progressive disease. Autopsy findings were consistent with previous biopsy results.

\section{Case 2}

A 63-year-old man diagnosed with a pNET following a workup of chronic diarrhea.

Investigation A $1.5 \mathrm{~cm}$ pancreatic neck mass with secondary hepatic lesions and celiac root lymphadenopathy was noted on initial CT examination and confirmed by a ${ }^{68}$ Gallium-DOTATATE PET-CT. The patient was diagnosed with well-differentiated NET grade 2 based on a biopsy of a metastatic hepatic lesion.

Treatment The patient was treated with Somatuline Autogel $120 \mathrm{mg} / \mathrm{month}$, and subsequently, due to progressive disease, he received four cycles of PRRT with ${ }^{177}$ Lutetium-DOTATATE. Due to further progression after a period of 3 years, another two cycles of ${ }^{177}$ Lutetium DOTATOC were administered, with an improvement in imaging studies.

Outcome and follow-up One year later the disease progressed again, as reflected by clinical deterioration and ${ }^{68}$ Gallium-DOTANOC PET-CT imaging (Fig. 2). In parallel, the patient developed recurrent episodes of severe hypoglycaemia, down to $1.2 \mathrm{mmol} / \mathrm{l}$.

Serum insulin and C-peptide levels in the fasting state revealed that the hypoglycemia of $1.2 \mathrm{mmol} / \mathrm{l}$ resulted from endogenous hyperinsulinism: insulin $144.2 \mathrm{pmol} / \mathrm{l}$ (normal range $=22-180$ ), and C-peptide $1006 \mathrm{pmol} / \mathrm{l}$ (normal range $=268-1274$ ). The patient was started on everolimus (10 mg/day) with resolution of the hypoglycemia and even the development of diabetes mellitus.

After a period of 8 months during which transarterial chemoembolization (TACE) of the metastases in the liver was performed, the patient developed recurrence of severe

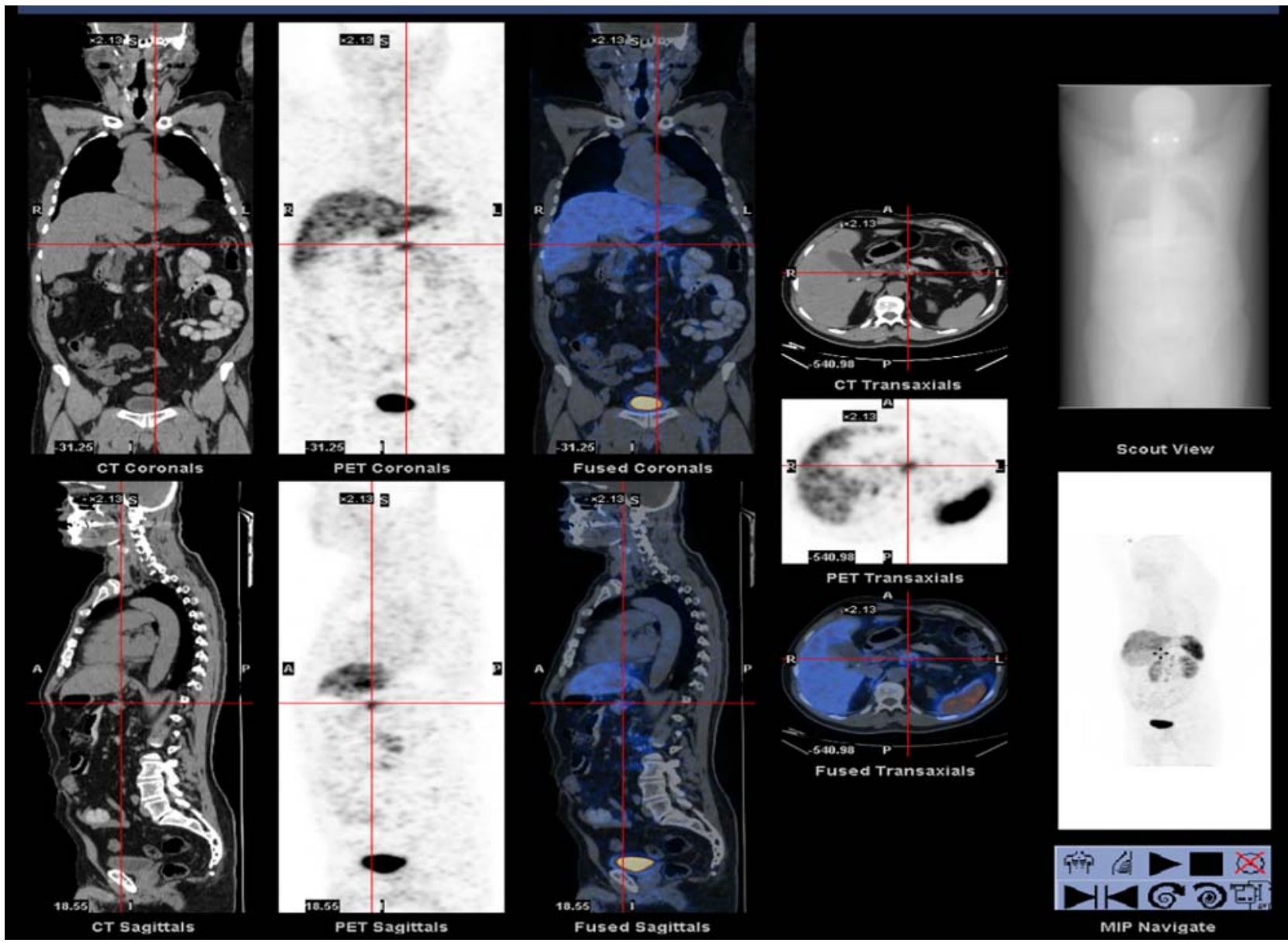

Figure 2

${ }^{68}$ Gallium-DOTANOC PET-CT of the second patient indicating diffuse involvement of liver, reflecting a salt-and-pepper appearance. 
hypoglycemic episodes and was treated with continuous nasogastric tube nutrition concurrently with diazoxide and dexamethazone.

\section{Case 3}

A 66-year-old man diagnosed with a metastatic gastrinoma following a workup of diarrhea.

Investigation A pancreatic mass with secondary hepatic lesions was noted on initial CT examination.

Treatment The patient was treated with $5-\mathrm{FU}$ and streptozotocin, which was stopped after a period of nine years due to kidney damage and progressive disease. Sandostatin LAR (octreotide LAR, Novartis) $60 \mathrm{mg} / \mathrm{month}$ was given. Subsequently, due to progressive disease he was referred for two PRRT treatments with ${ }^{90}$ Yttrium-DOTATOC and 3 years later with ${ }^{177}$ Lutetium-DOTATOC followed by disease stabilization. One year later the patient underwent the Whipple procedure due to obstructive jaundice. At the time of surgery liver metastases were also resected together with radio-frequency ablation. Following surgery, serum levels of gastrin and Chromogranin A normalized.

Outcome and follow-up One year later retroperitoneal lymph nodes spread was diagnosed; everolimus was started at a dosage of $10 \mathrm{mg} /$ day with ensuing disease stabilization. ${ }^{68}$ Gallium-DOTATATE PET-CT imaging revealed bilobar hepatic metastatic spread and a retroperitoneal $7.5 \mathrm{~cm}$ conglomerate of lymph nodes (Fig. 3). At this stage the patient developed recurrent episodes of severe symptomatic hypoglycemia of $1.9 \mathrm{mmol} / \mathrm{l}$, with inappropriately increased insulin $(750 \mathrm{pmol} / \mathrm{l}$, normal range $=22-180)$ and C-Peptide (1621 pmol/l, normal range $=268-1274$ ) levels, while still receiving everolimus.

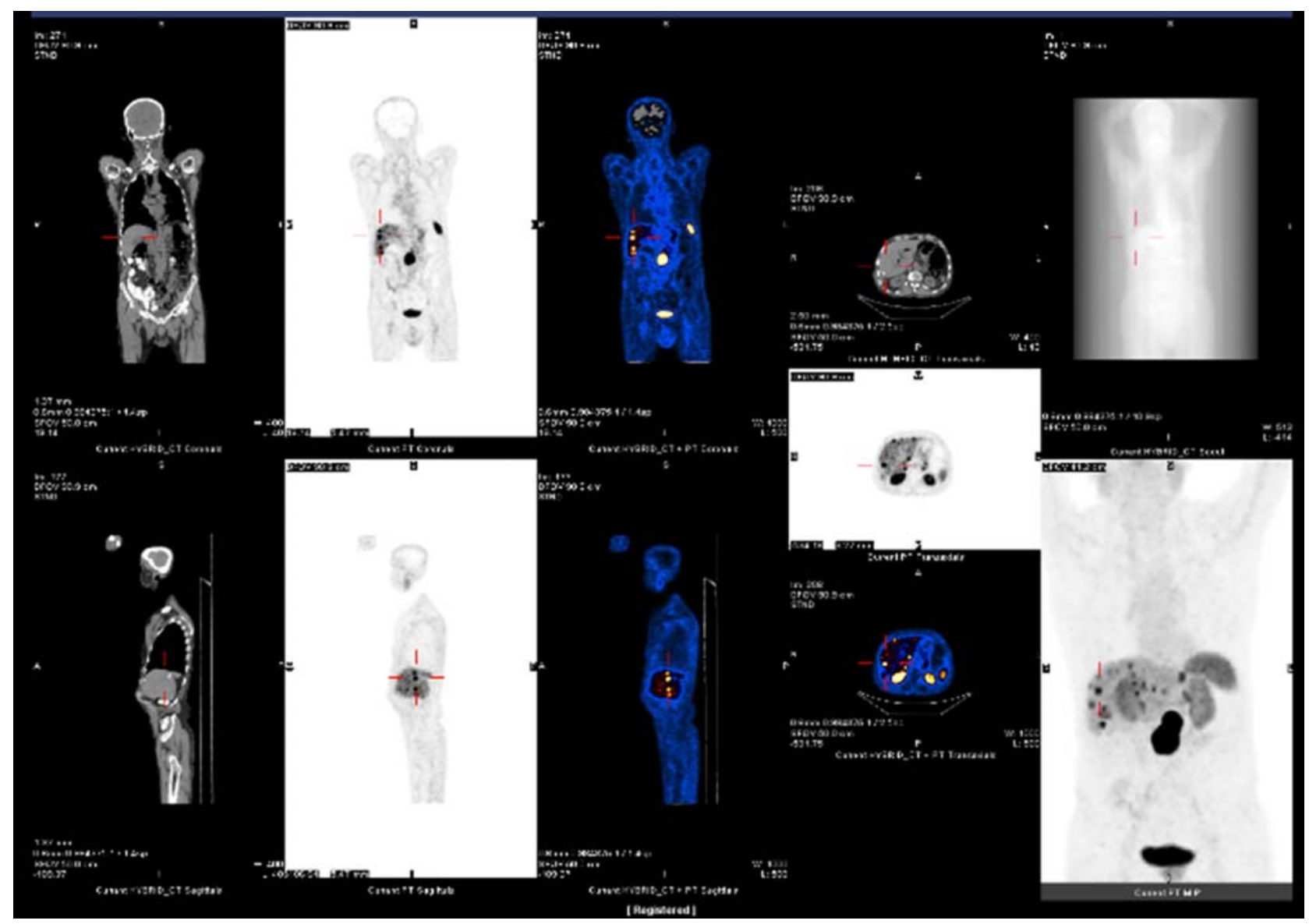

Figure 3

${ }^{68}$ Gallium-DOTATATE PET-CT of the third patient showing bilobar hepatic metastatic spread and a retroperitoneal conglomerate of lymph nodes. 


\section{Discussion}

In the present paper we describe a small series of three rare cases of pNETs, two initially nonfunctioning and one gastrinoma, which underwent phenotypic transformation to insulinoma, resulting in significant and life-threatening morbidity due to refractory hypoglycemia. To the best of our knowledge, this is the largest case series reported to date. pNETs are divided into nonfunctioning and functioning tumors, the latter being the minority. Functioning tumors are further defined, in accordance with the specific hormone secreted, as insulinoma, gastrinoma, glucagonoma, VIPomas, etc. The ability of pNET to 'gain' new functional phenotype has been reported previously, although very rare (4). These reports describe nonfunctioning pNETs that have transformed into an insulinoma, characterized by the appearance of repeated hypoglycemic episodes due to the excessive secretion of insulin (3). Very little is known regarding the mechanism of tumor transformation and gain of function. Interestingly, all of the cases described in the literature involved liver metastasis, suggesting a possible role for hepatic microenvironment in this process (3) (4). One possibility is that a subpopulation of insulin-producing cells that was clinically silent became prominent in the milieu of the liver. However, recent data done in pituitary NE cells suggested the existence of a pluripotent progenitor cell, which might be responsible for such plasticity in tumor phenotype (9). Refractory hypoglycemia remains an important cause of morbidity and mortality and contributes to the poor prognosis of these patients (3) (4). Given the rarity of patients presenting with pNETs with functional transformation, data about the treatment options in these patients is sparse and limited to rare case reports or expert opinion. Several treatment approaches have been tried in nonfunctioning pNETs with insulinoma transformation, such as systemic (somatostatin analogues, tyrosine kinase inhibitors such as sunitinib, mTOR inhibitors such as everolimus, or PRRT) or liver targeted (such as TACE). However, none of these proved to be highly effective in these cases.

Several lines of evidence support the role for mTOR in pNETs. Low protein expression of TSC2 and PTEN was associated with shortened progression-free survival and overall survival in patients with pNET. Tuberous sclerosis complex is a direct inhibitor of mTOR and takes a part in a genetic cancer syndrome associated with pNET (2) (10). These findings promoted the use of everolimus, an inhibitor of the mTOR signaling pathway, as a possible anti-tumor agent in these patients. Based on preclinical supporting data (11), the RADIANT-3 clinical study was conducted to assess the anti-proliferative effect of everolimus in pNETs patients: 410 patients with advanced pNETs have been enrolled and randomized to receive either everolimus or a placebo. Everolimus treatment was associated with median progression-free survival of 11 months, compared with 4.6 in the placebo arm, and with a 2.4-fold improvement in median progression-free survival compared with placebo (12).

Apart from its anti-tumor effect, everolimus can markedly improve the hypoglycemic control in insulinoma. This effect was shown to be mediated by a decrease in (pro)insulin secretion by the tumor (7). Interestingly, however, the anti-hypoglycemic effect of everolimus is also mediated by its effect on normal muscle tissue, with a marked reduction in glucose uptake, generating a state of insulin resistance (5) (7). In a retrospective series of 12 cases of metastatic insulinoma treated with everolimus, blood glucose levels were normalized in 11 patients. However, six of the patients had to discontinue the treatment due to cardiac or pulmonary adverse effects or tumor progression. At the time of the last follow-up, after a median of 7 months, seven patients experienced stable disease and three showed progression (8).

The effect of everolimus on hypoglycemia was also evident in two of our patients, in whom treatment with everolimus completely abolished the hypoglycemia events, leading to an improved quality of life for these patients. However, in both of these patients the disease progressed, and recurrent hypoglycemia reappeared during everolimus treatment. The third patient developed hypoglycemia while being treated with everolimus, and this exceptional escape from the hyperglycemic effect of everolimus has not been previously described in the literature.

In summary, the present small series of patients provides evidence on the ability of metastatic pNETs to gain new functional features. The development of functional phenotype bear grave prognosis. The awareness for the identification of such transformation is crucial for the treatment of these patients both in terms of providing accurate symptomatic support and possibly tailored therapy. It is possible that such gain of function might be more frequent than previously suspected, especially as some of these symptoms are not specific and might be attributed to other causes in a patient with an advanced malignant disease. Everolimus has emerged as a useful treatment for hypoglycemia in insulinoma patients and was also proven highly effective in the patients presented 
here, although it appears that, as disease progresses, the effect of everolimus on hypoglycemia wanes.

\section{Declaration of interest}

The authors declare that there is no conflict of interest that could be perceived as prejudicing the impartiality of the research reported.

\section{Funding}

This research did not receive any specific grant from any funding agency in the public, commercial or not-for-profit sector.

\section{Patient consent}

A consent form is submitted for the alive patient (one of the three).

\section{Author contribution statement}

A Nahmias is the resident who directly managed the presented patients. Prof. D J Gross and Dr S Grozinsky-Glasberg are senior doctors of the NET units and provided guidance. A Salmon is the accompanying oncologist.

\section{References}

1 Vinik AI 2014 Advances in diagnosis and treatment of pancreatic neuroendocrine tumors. Endocrine Practice 20 1222-1230. (doi:10.4158/ EP14373.RA)

2 Yao JC, Hassan M, Phan A, Dagohoy C, Leary C, Mares JE, Abdalla EK, Fleming JB, Vauthey JN, Rashid A et al 2008 One hundred years after "carcinoid": epidemiology of and prognostic factors for neuroendocrine tumors in 35,825 cases in the United States. Journal of Clinical Oncology 26 3063-3072. (doi:10.1200/JCO.2007.15.4377)

3 Vashi PG, Gupta D \& Dahlk S 2011 A unique case of a nonfunctional metastatic pancreatic neuroendocrine tumor transforming into an insulin-secreting tumor with an unusual clinical course. Pancreas $\mathbf{4 0}$ 781-784. (doi:10.1097/MPA.0b013e318212c42d)
4 Ohn JH, Kim YG, Lee SH \& Jung HS 2013 Transformation of nonofuctioning pancreatic neuroendocrine carcinoma cells into insulin producing cells after treatment with sunitinib. Endocrinology and Metabolism 28 149-152. (doi:10.3803/EnM.2013.28.2.149)

5 Meric-Bernstam F \& Gonzalez-Angulo AM 2009 Targeting the mTOR signaling network for cancer therapy. Journal of Clinical Oncology 27 2278-2287. (doi:10.1200/JCO.2008.20.0766)

6 Yao JC, Phan AT, Jehl V, Shah G \& Meric-Bernstam F 2013 Everolimus in advanced pancreatic neuroendocrine tumors: the clinical experience. Cancer Research 73 1449-1453. (doi:10.1158/0008-5472. CAN-12-3923)

7 Fiebrich HB, Siemerink EJ, Brouwers AH, Links TP, Remkes WS, Hospers GA \& de Vries EG 2011 Everolimus induces rapid plasma glucose normalization in insulinoma patients by effects on tumor as well as normal tissues. Oncologist 16 783-787. (doi:10.1634/ theoncologist.2010-0222)

8 Bernard V, Lombard-Bohas C, Taquet MC, Caroli-Bosc FX, Ruszniewski P, Niccoli P, Guimbaud R, Chougnet CN, Goichot B, Rohmer V et al 2013 Efficacy of everolimus in patients with metastatic insulinoma and refractory hypoglycemia. European Journal of Endocrinology/European Federation of Endocrine Societies 168 665-674. (doi:10.1530/EJE-12-1101)

9 Florio T 2011 Adult pituitary stem cells: from pituitary plasticity to adenoma development. Neuroendocrinology 94 265-277. (doi:10.1159/ 000330857)

10 Barbieri F, Albertelli M, Grillo F, Mohamed A, Saveanu A, Barlier A Ferone D \& Florio T 2014 Neuroendocrine tumors: insights into innovative therapeutic options and rational development of targeted therapies. Drug Discovery Today 19 458-468. (doi:10.1016/j.drudis. 2013.10.015)

11 Grozinsky-Glasberg S, Franchi G, Teng M, Leontiou CA, Ribeiro de Oliveira A, Jr, Dalino P, Salahuddin N, Korbonits M \& Grossman AB 2008 Octreotide and the mTOR inhibitor RAD001 (everolimus) block proliferation and interact with the Akt-mTOR-p70S6K pathway in a neuro-endocrine tumour cell line. Neuroendocrinology 87 168-181. (doi:10.1159/000111501)

12 Taha C, Liu Z, Jin J, Al-Hasani H, Sonenberg N \& Klip A 1999 Opposite translational control of GLUT1 and GLUT4 glucose transporter mRNAs in response to insulin. Role of mammalian target of rapamycin, protein kinase B, and phosphatidylinositol 3-kinase in GLUT1 mRNA translation. Journal of Biological Chemistry 274 33085-33091. (doi:10.1074/ jbc.274.46.33085)

Received in final form 21 May 2015 Accepted 29 May 2015 